dessen Lektüre wohl die meisten Studierenden aussteigen. Darum erübrigt sich auch die Besprechung der Kurse 2 und 3.

Rudolf Stöber

\section{Uta Rußmann}

\section{Agenda Setting und Internet}

Themensetzung im Spannungsfeld von Onlinemedien und sozialen Netzwerken

München: Fischer, 2007. - 367 S.

ISBN 978-3-88927-435-9

Die stetige Zunahme von Kommunikationskanälen und die Individualisierung der Nutzungsmuster, mit denen diese in Anspruch genommen werden, konfrontiert die Medienwirkungsforschung mit zwei zentralen Herausforderungen. Auf der theoretischen Ebene gilt es, die Haltbarkeit etablierter Ansätze, die unter den Bedingungen der Kanalknappheit entwickelt wurden, zu überprüfen und gegebenenfalls zu modifizieren oder durch alternative Konzepte zu ersetzen. Auf der empirischen Ebene gilt es, Analysemodelle und -verfahren zu entwickeln, die komplex genug sind, um der entstandenen Unübersichtlichkeit und Interdependenz auf der Seite der unabhängigen Variablen gerecht zu werden. Die Dissertation von Uta Rußmann erhebt den Anspruch, beiden Anforderungen gerecht werden zu wollen. Ihr konkretes Ziel ist es, „die Tragfähigkeit der Agenda-Setting-Hypothese im gegenwärtigen Medienwandel“ $\mathrm{zu}$ testen und damit einen theoretischen wie methodischen „Beitrag zur Weiterentwicklung der Medienwirkungsforschung" (S. 17) zu leisten. Erreicht werden soll dies zum einen durch die Entwicklung eines theoretischen Verständnisses für das Zusammenspiel „von Themensetzungs- und Themenstrukturierungsprozessen, individueller Informationsrezeption sowie Onlineangeboten im Medien-Mix der traditionellen Massenmedien vor dem Hintergrund realer, sozialer Kommunikationsnetzwerke“ (S. 17). Die empirische Überprüfung der entsprechenden Annahmen soll mit Hilfe eines Agenda-Setting-Designs realisiert werden, „welches jedoch das jeweilige gesellschaftliche Umfeld einbezieht, um so vor allem Aussagen über die Auswirkungen von Agenda-Setting-Effekten in realen Situationen zu treffen" (S. 195).

Dieser etwas umständlich formulierte, aber zweifellos stolze Anspruch erweist sich im Folgenden als schwere Hypothek. Im Theorieteil stellt die Autorin zwar ihre Fähigkeit unter Beweis, wissenschaftliche Argumentationen aufarbeiten und empirische Erträge zusammenfassen zu können. Gleichzeitig ist sie jedoch nur bedingt in der Lage, die Selektion der berücksichtigten Ansätze nachvollziehbar zu begründen sowie das üppige Material zu bündeln, zueinander in Beziehung zu setzen und an eine durchgehende Argumentation oder zumindest an eine zentrale Fragestellung zu binden. Kapitel 10 stellt zwar eine theoretische Zusammenfassung in Aussicht und präsentiert dazu zwei Forschungsdesigns. Diese werden allerdings kaum kommentiert und lassen sich bestenfalls punktuell aus den vorangegangenen Kapiteln ableiten. Die unzureichende Verdichtung der theoretischen Argumentation findet ihre Fortsetzung in einem Katalog von 21 forschungsleitenden Fragestellungen, acht forschungsleitenden Annahmen und einer Visualisierung der angestrebten empirischen Untersuchung in Form eines Strukturgleichungsmodells (!) mit insgesamt 17 Einflussfaktoren. Die empirische Untersuchung, die einerseits als Exploration (S. 197) und andererseits als Überprüfung des Analysemodells (S. 198) angekündigt wird, hat es schwer, dieser Komplexität auch nur ansatzweise gerecht zu werden. Stattdessen beschränkt sie sich im Wesentlichen darauf, die Daten einer im Kontext der österreichischen Präsidentschaftswahlen im Jahre 2004 durchgeführten Befragung von 29 Offlinern und 76 Onlinern per Kreuztabelle oder per Mittelwertvergleich zu kontrastieren. Die Zahl der dabei berücksichtigten Variablen ist zwar beachtlich, die Analyse reicht aber kaum über das Niveau einer Deskription von Unterschieden hinaus. Schwer nachvollziehbar ist dabei zudem, dass die Themenagenda des Publikums als zentrales Konstrukt der Agenda-Setting-Forschung mit fortschreitender Analyse mehr und mehr in den Hintergrund rückt, um schließlich bei der als Makroanalyse (Kapitel 17) bezeichneten Untersuchung egozentrierter Netzwerke völlig aus dem Blick zu verschwinden.

Man könnte der Autorin angesichts ihres eigenen Anspruchs zugute halten, auf hohem Niveau gescheitert zu sein. Wären da nicht weitere methodische Unzulänglichkeiten (ungenügende Transparenz der inhaltsanalytischen Vorgehensweise, Verzicht auf Reliabilitätstests, Mittelwertvergleiche bei dichotomen 
Variablen) und formale Defizite (Gliederungsfehler, Verzicht auf Tabellen- und Abbildungsverzeichnis, lieblose bis unleserliche Grafiken), mit denen die Arbeit auch weniger ambitionierte Erwartungen unterläuft.

Angesichts der genannten Vorbehalte ist mit Blick auf die gewonnenen Befunde kaum zu beurteilen, ob etwa der behauptete Nachweis einer Beeinträchtigung der massenmedialen Thematisierungsfunktion durch die Onlinenutzung tatsächlich erbracht wurde. Was die Arbeit stattdessen belegt, ist erstens die Tatsache, dass theoretischer Fortschritt nicht durch die additive Kumulation von möglichst vielen verschiedenen Ansätzen erreicht wird, und dass zweitens die Komplexität der empirischen Realität nur bedingt erfasst werden kann, indem man möglichst viele verschiedene abhängige Dimensionen ohne systematische Kontrolle von Drittvariablen über die immer gleiche Dichotomie bricht.

Mirko Marr

\section{Tobias Scheel}

\section{Die staatliche Festsetzung der Rundfunk- gebühr}

Rechtliche Kriterien und Grenzen der Gestaltungsmacht der Länder im Verfahren zur Festsetzung der Rundfunkgebühr

Berlin: Duncker \& Humblot 2007. - 241 S.

ISBN 978-3-428-12323-0

Die zu besprechende Abhandlung, die der Juristischen Fakultät der Friedrich-AlexanderUniversität Erlangen-Nürnberg als Dissertation vorgelegen hat, befasst sich mit interessanten und - wie jüngere Entwicklungen gezeigt haben - praktisch überaus bedeutsamen Fragen des Rundfunkgebührenrechts. In insgesamt sechs Teilen beleuchtet Scheel intensiv und umfassend die Reichweite des Entscheidungsspielraumes der Länder bei der Festsetzung der Rundfunkgebühr.

$\mathrm{Zu}$ Beginn zeichnet der Autor die historische Entwicklung der Rundfunkgebühr sowie das Verfahrens ihrer Festsetzung nach. Er geht dabei anschaulich auf die Umstände der einzelnen Gebührenerhöhungen vor allem im Kontext mit den zum jeweiligen Zeitpunkt vorherrschend diskutierten politischen und rechtlichen Fragestellungen ein. Den Schlusspunkt der dargestellten Entwicklung bilden die
Auseinandersetzungen um die Gebührenerhöhung im 8. Rundfunkänderungsstaatsvertrag, mit dem die Länder vom Gebührenvorschlag der Kommission zur Überprüfung und Ermittlung des Finanzbedarfs der Rundfunkanstalten (KEF) nach unten abwichen. Das darauf folgende zweite Gebührenurteil vom 11. September 2007, in dem das BVerfG eine Verletzung der Rundfunkfreiheit der Beschwerde führenden öffentlich-rechtlichen Rundfunkanstalten feststellte, konnte keine Berücksichtigung mehr finden (Stand der Dissertation: Februar 2006).

Es folgen die Darstellung der verfassungsrechtlichen Rahmenbedingungen in Art. 5 Abs. 1 S. 2 GG sowie ein Überblick über die europarechtliche Beihilfeproblematik. Der Autor geht auf die Interpretation der Rundfunkfreiheit durch das BVerfG als „dienende Freiheit" und den damit verbundenen Auftrag an den Gesetzgeber ein, eine positive Rundfunkordnung zu schaffen. Im Kontext der verfassungsrechtlich gebotenen funktionsgerechten Finanzierung des öffentlich-rechtlichen Rundfunks weist Scheel besonders darauf hin, dass dem Staat neben dieser Finanzierungsverantwortung auch ein Wächteramt hinsichtlich der monetären Rezipienteninteressen zukommt.

Im dritten Teil untersucht Scheel das geltende Verfahren der Gebührenfestsetzung. Das strukturelle Dilemma zwischen der Programmautonomie der Anstalten sowie der Programmneutralität der Finanzierung einerseits und der Bestimmung des Funktionsauftrages durch den Gesetzgeber sowie der Programmakzessorietät der Finanzierung andererseits wird von Scheel ebenso präzise dargelegt wie das vom BVerfG zur Lösung dieses Dilemmas im ersten Gebührenurteil entwickelte dreistufige Verfahren zur Festsetzung der Rundfunkgebühr, das in Folge dieser Entscheidung 1996 im 3. Rundfunkänderungsstaatsvertrag seine einfachgesetzliche Umsetzung erfuhr.

Im anschließenden vierten Teil der Arbeit beschäftigt sich Scheel intensiv und äußerst reflektiert mit der dritten Stufe des Verfahrens, der endgültigen Festlegung der Gebührenhöhe durch die Landesgesetzgeber. Es geht dabei also um die Frage, ob und inwieweit dem KEF-Votum Bindungswirkung zukommt. Die Möglichkeit einer Abweichung vom Gebührenvorschlag der KEF erschöpft sich nach Ansicht des BVerfG im Wesentlichen in den Gesichtspunkten des Informationszugangs und 